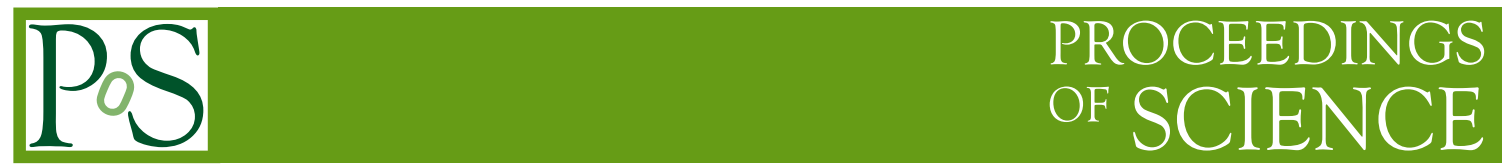

\title{
Re-assessment of the nucleon Boer-Mulders function
}

\author{
Elliot Leader*t \\ Author affiliation \\ Imperial College London E-mail: e. Leaderdimperial.ac.uk
}

The BM functions in the literature, based on certain assumptions about the proportionality between the BM and the Sivers functions, are shown to be incorrect. New relations which are consistent with data are studied.

XXVII International Workshop on Deep-Inelastic Scattering and Related Subjects - DIS2019

8-12 April, 2019

Torino, Italy

\footnotetext{
*Speaker.

${ }^{\dagger}$ in collaboration with E. Christova, M. Stoilov and D. Strozik-Kotlorz
} 
The Sivers function encodes the correlation between the spin $\boldsymbol{S}_{N}$ of a polarized nucleon, the nucleon momentum $\boldsymbol{P}_{N}$ and the quark (transverse) momentum $\boldsymbol{k}_{q}$ via a term proportional to $\boldsymbol{S}_{N}$. $\left(\boldsymbol{k}_{q} \times \boldsymbol{P}_{N}\right)$. The Boer-Mulders (BM) function encodes the correlation between the quark spin $\boldsymbol{s}_{q}$, its momentum and the momentum of an unpolarized nucleon via a term proportional to $\boldsymbol{s}_{q} \cdot\left(\boldsymbol{k}_{q} \times \boldsymbol{P}_{N}\right)$. Information about these functions is extracted from data on various asymmetries in SIDIS reactions involving unpolarized leptons colliding with either unpolarized or tranversely polarized nucleons, and with monitoring of the azymuthal angle $\phi_{h}$ of the produced hadron $h$ :

$$
\begin{aligned}
& l_{\mathrm{U}}+N_{\mathrm{T}} \rightarrow l^{\prime}+h\left(\phi_{h}\right)+X \\
& l_{\mathrm{U}}+N_{\mathrm{U}} \rightarrow l^{\prime}+h\left(\phi_{h}\right)+X
\end{aligned}
$$

Because of the scarcity of data, it has been customary in the literature to assume that

$$
(\mathrm{BM})_{q}=\lambda_{q}(\mathrm{Siv})_{q}
$$

for each quark and antiquark and to evaluate the $\lambda_{q}$ by fitting the data. But this is incorrect theoretically. It leads to a gluon contribution to the flavour non-singlet $(\mathrm{BM})_{(q-\bar{q})}$ unless

$$
\lambda_{q}=\lambda_{\bar{q}}
$$

which is not the case.

In a recent paper [W] we explored a variant of the above assumption, which, however, is theoretically acceptable. Namely, to avoid the complication of gluons we worked with asymmetry differences

$$
A^{h-\bar{h}} \equiv \frac{\Delta \sigma^{h}-\Delta \sigma^{\bar{h}}}{\sigma^{h}-\sigma^{\bar{h}}} .
$$

which involve only valence contributions $q_{V}$. We utilized deuteron data only and tried the analogue of Eq. (1) for the relevant valence quark combinations

$$
(\mathrm{BM})_{Q_{V}}=\lambda_{Q_{V}}(\mathrm{Siv})_{Q_{V}}
$$

where

$$
Q_{V}=u_{V}+d_{V}
$$

This led to two relations between the unpolarized asymmetries $A_{U U}^{\cos \phi_{h}, h-\bar{h}}$ and $A_{U U}^{\cos 2 \phi_{h}, h-\bar{h}}$ and the polarized Sivers asymmetry $A_{U T}^{S i v, h-\bar{h}}$. For deuterons we had:

$$
\begin{gathered}
A_{U U}^{\cos \phi_{h}, h-\bar{h}}(x)=\Phi(x)\left\{C_{B M}^{h} A_{U T}^{S i v, h-\bar{h}}(x)+C_{C a h n}^{h}\right\} \\
A_{U U}^{\cos 2 \phi_{h}, h-\bar{h}}(x)=\hat{\Phi}(x)\left\{\hat{C}_{B M}^{h} A_{U T}^{S i v, h-\bar{h}}(x)+\frac{M_{d}^{2}}{\left\langle Q^{2}\right\rangle} \hat{C}_{C a h n}^{h}\right\}
\end{gathered}
$$

where $\Phi(x)$ and $\hat{\Phi}(x)$ are known functions. The $C_{C a h n}^{h}, \hat{C}_{C a h n}^{h}, C_{B M}^{h}, \hat{C}_{B M}^{h}$ are constants, which depend on the mean $\left\langle k_{\perp}^{2}\right\rangle$ of transverse momentum dependent PDFs and on the mean $\left\langle p_{\perp}^{2}\right\rangle$ of transverse momentum dependent FFs, assumed in the present literature to be flavour independent, and on $M_{C}$ and $M_{S}$, which determine the transverse momentum dependence of the Collins and 
Sivers functions respectively. Several different sets of values for these parameters exist in the literature,ranging from 0.18 to 0.61 for $\left\langle k_{\perp}^{2}\right\rangle$ and from 0.12 to 0.20 for $\left\langle p_{\perp}^{2}\right\rangle$. We used COMPASS deuteron data [Q, B] for the asymmetries and found that our relations were well satisfied for the sets of values

$$
\left\langle k_{\perp}^{2}\right\rangle_{P D F}=0.18 \text { or } 0.25 \text { with }\left\langle p_{\perp}^{2}\right\rangle_{F F}=0.20 .
$$

We published our results (Christova, Leader and Stoilov: CLS) in 2018 [四].

We were shocked to learn later that the COMPASS asymmetries do NOT correspond to the asymmetries $A_{U U}^{\cos \phi_{h}}, A_{U U}^{\cos 2 \phi_{h}}$ in the literature! The symbols we used are standard ones in the literature, and are defined for an arbitrary angular weight function in Eq. (79) of [四], which article, in turn, is based on Eq. (2.7) of the Trento Convention article [5]. The asymmetries are defined logically so that their values lie between -1 and 1 .

We therefore took COMPASS to court, (at least in our imagination), accused of disseminating potentially misleading information. COMPASS pleaded NOT GUILTY on two grounds:

1) The COMPASS symbols were $A_{\cos \phi_{h}}^{U U}$ and NOT $A_{U U}^{\cos \phi_{h}}$ etc. The Judge dismissed this as irrelevant!

2) COMPASS claimed we should have read their Eq. (1) more carefully. It reads:

“..........given in the one-photon exchange approximation [17] by:

$$
d \sigma=\sigma_{0}\left(1+\varepsilon_{1} A_{\cos \phi_{h}}^{U U} \cos \phi_{h}+\ldots\right) "
$$

The Judge commented that he was unable to find this equation in reference [17]!

Nonetheless, and to our astonishment, the Judge pronounced COMPASS NOT GUILTY, but asked them to take more care to warn readers of potential misunderstandings in future.

We therefore took the case to the Court of Appeal, pointing out:

1) that the Trento Convention for single spin asymmetries had been established in 2004 [6]

2) that the conventions had been reiterated in a major review in 2008 [ [0]

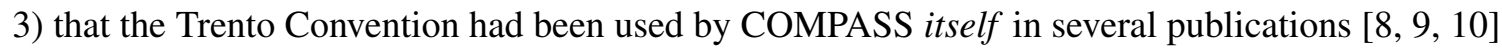
and by HERMES []]

To our amazement the Court of Appeal upheld the decision of the lower court!

Hence my intention today was to announce that our results in PR D97, 056018 (2018) are TOTAL RUBBISH.

However MIRACLES DO HAPPEN!

With the COMPASS definitions of the asymmetries the incorrect (CLS) and correct (COMP) version of the relations Eq. (囚, Ø) for deuterons read:

$$
\begin{gathered}
\left.A_{U U}^{\cos \phi_{h}, h-\bar{h}}(x)\right|_{\text {COMP }}=\Phi_{C L S, C O M P}\left\{C_{B M}^{h} A_{U T, d}^{S i v, h-\bar{h}}(x)+C_{C a h n}^{h}\right\}, \\
\left.A_{U U}^{\cos 2 \phi_{h}, h-\bar{h}}(x)\right|_{\text {COMP }}=\hat{\Phi}_{C L S, C O M P}\left\{\hat{C}_{B M}^{h} A_{U T, d}^{S i v, h-\bar{h}}(x)+\frac{M_{d}^{2}}{\left\langle Q^{2}\right\rangle} \hat{C}_{C a h n}^{h}\right\} .
\end{gathered}
$$

THE MIRACLE: The functions $\Phi(x), \hat{\Phi}(x)$ are ratios of polynomials. Remarkably as seen in FIG. 1, comparison of $\Phi_{C L S}$ with $\Phi_{C O M P}$ and $\hat{\Phi}_{C L S}$ with $\hat{\Phi}_{C O M P}$ shows that they are essentially equal in the kinematical region of interest.

The unexpected conclusion is then that the results of PR D97, 056018 (2018) are correct! 


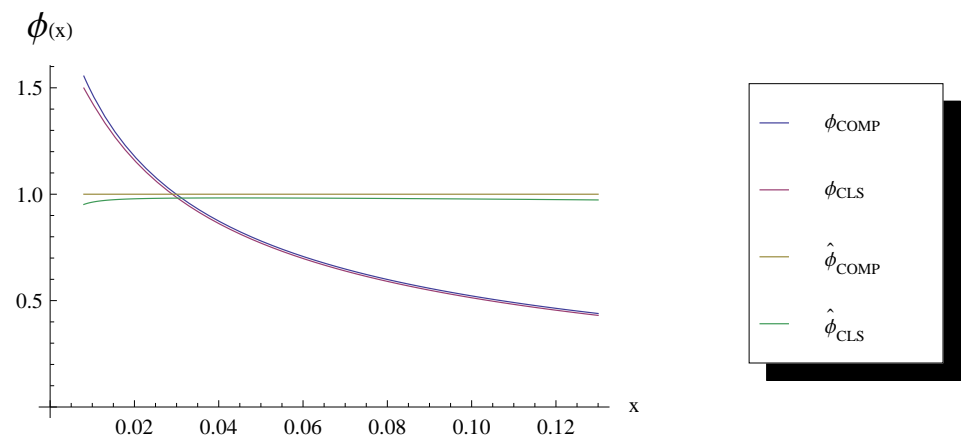

Figure 1: Comparison of $\Phi_{C L S}$ with $\Phi_{C O M P}$ and $\hat{\Phi}_{C L S}$ with $\hat{\Phi}_{C O M P}$.

Here, then, is a summary of the PRD conclusions of CLS: two independent tests of the assumption

$$
(\mathrm{BM})_{Q_{V}} \propto(\text { Sivers })_{Q_{V}}
$$

were made using the COMPASS deuteron SIDIS data on the difference asymmetries $A_{U U, d}^{\cos \phi_{h}, h^{+}-h^{-}}(x)$, $A_{U U, d}^{\cos 2 \phi_{h}, h^{+}-h^{-}}(x)$ and $A_{U T, d}^{S i v, h^{+}-h^{-}}(x)$. Both tests are consistent with this assumption in the kinematic interval $x=[0.014,0.13]$. The results are very sensitive to the average transverse momentumsquared, $\left\langle k^{2}\right\rangle$ and $\left\langle p^{2}\right\rangle$ of the unpolarized momentum dependent PDFs and FFs, respectively, with clear preference for the old experimental values, in units of $\mathrm{GeV}^{2}:\left\langle k^{2}\right\rangle \approx 0.18$, or $\left\langle k^{2}\right\rangle \approx 0.25$, $\left\langle p^{2}\right\rangle \approx 0.20$, and the values $M_{S}^{2}=0.34$ or $M_{S}^{2}=0.19$ and $M_{C}^{2}=0.91$ for the parameters controlling the momentum dependence of the Sivers and Collins functions respectively.

Our results suggested the previous extraction of the BM function by Barone, Melis and Prokudin (BMP) [ए2] might be unreliable. We are now (with D. Strozik-Kotlorz: CLS-K) in the pro-

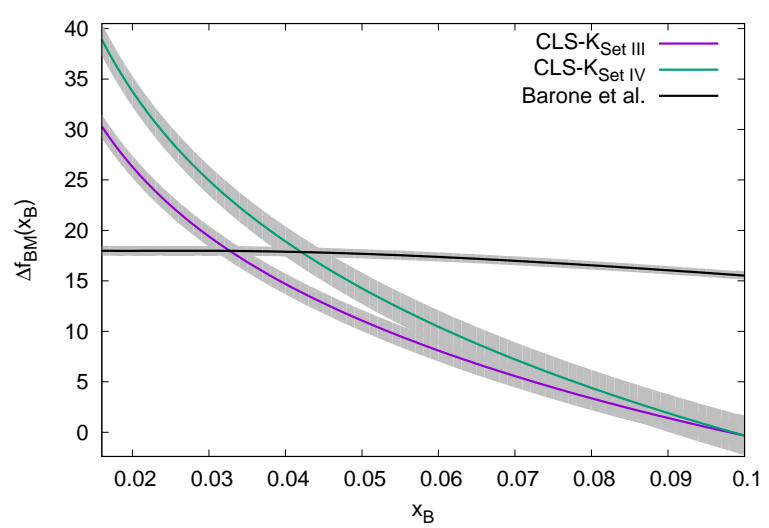

Figure 2: Comparison between the $\mathrm{BM}_{Q_{V}}$ function, constructed from the Barone et al results, and the $\mathrm{BM}_{Q_{V}}$ function extracted directly from the difference asymmetries.

cess of studying the extraction of the VALENCE BM function directly from $A_{U U, d}^{\cos \phi_{h}, h^{+}-h^{-}}(x)$, and $A_{U U, d}^{\cos 2 \phi_{h}, h^{+}-h^{-}}(x)$, without making any assumptions about its relation to the Sivers function. We have 
used the BMP $(\mathrm{BM})_{q}$ and $(\mathrm{BM})_{\bar{q}}$ results to compute the BMP version of $(\mathrm{BM})_{Q_{V}}$ and compared it to our preliminary results, for two choices of PDF and FF average transverse momenta, as shown in Fig. 2. It is seen that the BMP version is significantly different from ours, supporting our earlier conclusion that the BMP BM functions, which, as explained earlier, are based on a theoretically unacceptable assumption, are incorrect.

We expect to have final results on the valence BM function very soon. Also to have a more definitive statement about the sensitivity to the transverse momentum dependence of the PDFs and FFs.

\section{Acknowledgements}

E.C. and M.S. thank the Bulgarian Science Foundation for Grant 08-17/2016. DSK acknowledges the support of the Bogoliubov-Infeld Program and the Collaboration Program JINRBulgaria.

\section{References}

[1] E. Christova, E. Leader, and M. Stoilov, Phys. Rev. D97, 056018 (2018), arXiv:1705.10613 [hep-ph].

[2] C. Adolph et al. (COMPASS), Nucl. Phys. B886, 1046 (2014), arXiv:1401.6284 [hep-ex].

[3] E. S. Ageev et al. (COMPASS), Nucl. Phys. B765, 31 (2007), arXiv:hep-ex/0610068.

[4] M. Anselmino, M. Boglione, U. DŠAlesio, S. Melis, F. Murgia, E. R. Nocera, and A. Prokudin, Phys. Rev. D83, 114019 (2011), arXiv:1101.1011 [hep-ph].

[5] A. Bacchetta, M. Diehl, K. Goeke, A. Metz, P. J. Mulders, and M. Schlegel, JHEP 02, 093 (2007), arXiv:hep-ph/0611265.

[6] A. Bacchetta, U. D’Alesio, M. Diehl, and C. A. Miller, Phys. Rev. D70, 117504 (2004), arXiv:hep-ph/0410050.

[7] D'Alesio, U. and Murgia, F., Prog. Part. Nucl. Phys.61 (2008), 394, arXiv:0712.4328, hep-ph

[8] F. Bradamante (COMPASS), Proceedings, 17th International Spin Physics Symposium (SPIN06): Kyoto, Japan, October 2-7, 2006, AIP Conf. Proc. 915, 513 (2007), arXiv:hep- ex/0702007.

[9] W. Kafer (COMPASS), in Transversity 2008: 2nd International Work- shop on Transverse Polarization Phenomena in Hard Processes Ferrara, Italy, May 28-31, 2008 (2008) pp. 169-176, arXiv:0808.0114 [hep-ex].

[10] A. Bressan (COMPASS), in Proceedings, 17th International Workshop on Deep-Inelastic Scattering and Related Subjects (DIS 2009): Madrid, Spain, April 26-30, 2009 (2009) p. 211, arXiv:0907.5511 [hep-ex].

[11] F. Giordano and R. Lamb (HERMES), Proceedings, 18th International Spin Physics Sym- posium (SPIN 2008): Charlottesville, USA, October 6-11, 2008, AIP Conf. Proc. 1149, 423 (2009), arXiv:0901.2438 [hep-ex].

[12] V. Barone, S. Melis, and A. Prokudin, Phys. Rev. D81, 114026 (2010), arXiv:0912.5194 [hep-ph]. 